\title{
Digital signal processing methods for impedance microfluidic cytometry
}

\author{
Tao Sun · Cees van Berkel • Nicolas G. Green · \\ Hywel Morgan
}

Received: 1 April 2008/ Accepted: 28 May 2008

(C) Springer-Verlag 2008

\begin{abstract}
Impedance microfluidic cytometry is a noninvasive, label-free technology that can characterize the dielectric properties of single particles (beads/cells) at high speed. In this paper we show how digital signal processing methods are applied to the impedance signals for noise removal and signal recovery in an impedance microfluidic cytometry. Two methods are used; correlation to identify typical signals from a particle and for a noisier environment, an adaptive filter is used to remove noise. The benefits of adaptive filtering are demonstrated quantitatively from the correlation coefficient and signal-to-noise ratio. Finally, the adaptive filtering method is compared to the Savitzky-Golay filtering method.
\end{abstract}

Keywords Microfluidics - Single cell analysis ·

Impedance spectroscopy · Correlation · Adaptive filtering

\section{Introduction}

Single cell analysis requires precise manipulation and characterization to be performed in micro scale devices

\footnotetext{
T. Sun · N. G. Green · H. Morgan $(\bowtie)$

Nanoscale Systems Integration Group,

School of Electronics and Computer Science,

University of Southampton,

Southampton SO17 1BJ, UK

e-mail: hm@ecs.soton.ac.uk

T. Sun

e-mail: ts5@ecs.soton.ac.uk

C. van Berkel

Philips Research Laboratory,

Cross Oak Lane, Redhill,

Surrey RH1 5HA, UK
}

using Lab-on-a-Chip (LOC) technology. Dielectric or impedance spectroscopy has been used to discriminate cells on the basis of size and dielectric properties. Characterization of the dielectric properties of particles can be performed in two ways, using AC electrokinetic techniques (Morgan and Green 2003) or electrical impedance methods (Morgan et al. 2007). Impedance spectroscopy has been implemented on a microfluidic chip, providing a high speed method of characterising the dielectric properties of different micron-sized particles.

The first cytometer capable of measuring the electrical properties of a single particle was developed by Coulter (1956). The device measured the DC resistance (or low frequency impedance) between two electrically isolated fluid-filled chambers as particles passed through a small connecting orifice. For a fixed sized orifice, the change in electrical current is used to count and size the cells. Single particle impedance measurement can be categorized as stationary or dynamic (flow-cytometry) measurements. Recently, Cho et al. (2006) fabricated twin microcantilever arrays in microchannels for measuring the impedance of normal and abnormal red blood cells. Malleo et al. (2007) developed a microfluidic chip to hydrodynamically capture single cells and analyse the kinetics of cell response to Streptolysin-O. Jang and Wang (2007) fabricated a polydimethylsiloxane (PDMS) channel with pillars to capture a single cell and measured the impedance. Wang et al. (2008) used a metal oxide semiconductor field effect transistor (MOSFET) to detect the resistive pulse induced by CD4+ T-lymphocytes in a PDMS channel.

In a miniaturized flow-cytometry, particles flow through a microfluidic channel in which microelectrodes are integrated. Ayliffe et al. (1999) developed a micro-impedance measurement device with integrated electrodes to measure the impedance of femtoliter quantities of ionic solutions 
and single biological cells. Following on this work, impedance micro-cytometers capable of measuring hundreds of individual cells were developed (Gawad et al. 2001, 2004; Cheung et al. 2005; Holmes et al. 2005; Morgan et al. 2006, 2007; Sun et al. 2007a). An example of a cytometer is shown in Fig. 1. Microelectrodes are patterned on top and bottom glass substrates. The size of the electrodes is typically $20-40 \mu \mathrm{m}$ providing optimal sensitivity (Sun et al. 2007a). The microfluidic channel is made from a thick resist, giving a typical channel width and height of $20 \mu \mathrm{m}$. Two pairs of electrodes are used to define the impedance measurement area, enabling a differential impedance measurement to be performed. As particles flow through the electrodes, the differential signal gives one positive and one negative peak (Fig. 1). Impedance information is extracted from the peaks of this bipolar signal. The velocity of the particle is determined from the time difference between the two peaks (ms).

In almost all microfluidic-based single cell analysis systems, effort has centred on fabricating novel designs of micro-device and developing new detection techniques, but there has been little attempt at developing ways of extracting meaningful data from the often noisy signals using signal processing. In this paper, we apply two different digital signal processing methods to single particle impedance signals obtained using a microfluidic cytometer (Fig. 1), and demonstrate the benefits for particle analysis.

\section{Micro-impedance measurement system}

Two different measurement systems have been developed, a discrete frequency system, and a time domain analysis method called maximum length sequence (MLS) analysis.

\subsection{AC discrete frequency system}

A schematic diagram of the discrete frequency system is shown in Fig. 2a. Two AC excitation signals (low and high frequency) are mixed and simultaneously applied to the one pair of electrodes (in Fig. 2a). The current from the detection and reference volumes is measured using a differential amplifier. The signal is demodulated with a lockin amplifier giving the in-phase and out-of phase signals for each frequency. Data acquisition and analysis is performed using a PC. Full details of this system have been reported previously (Gawad et al. 2001, 2004; Cheung et al. 2005; Holmes et al. 2005; Morgan et al. 2006, 2007).

This method gives good signal-to-noise ratio (SNR), because the energy in the excitation signal (mixed) is concentrated at the discrete frequencies and the lock-in amplifiers rejects all other noise sources. The major drawback is that the number of measured frequencies is restricted because each frequency requires a lock-in amplifier for signal demodulation. An analog applicationspecific integrated circuit (ASIC) can be used to
Fig. 1 Diagram showing the design of a typical impedance micro-cytometer used for single cell analysis. Two pairs of parallel (facing) electrodes are used to perform a differential impedance measurement. A cell flows through the measurement area producing a double peak

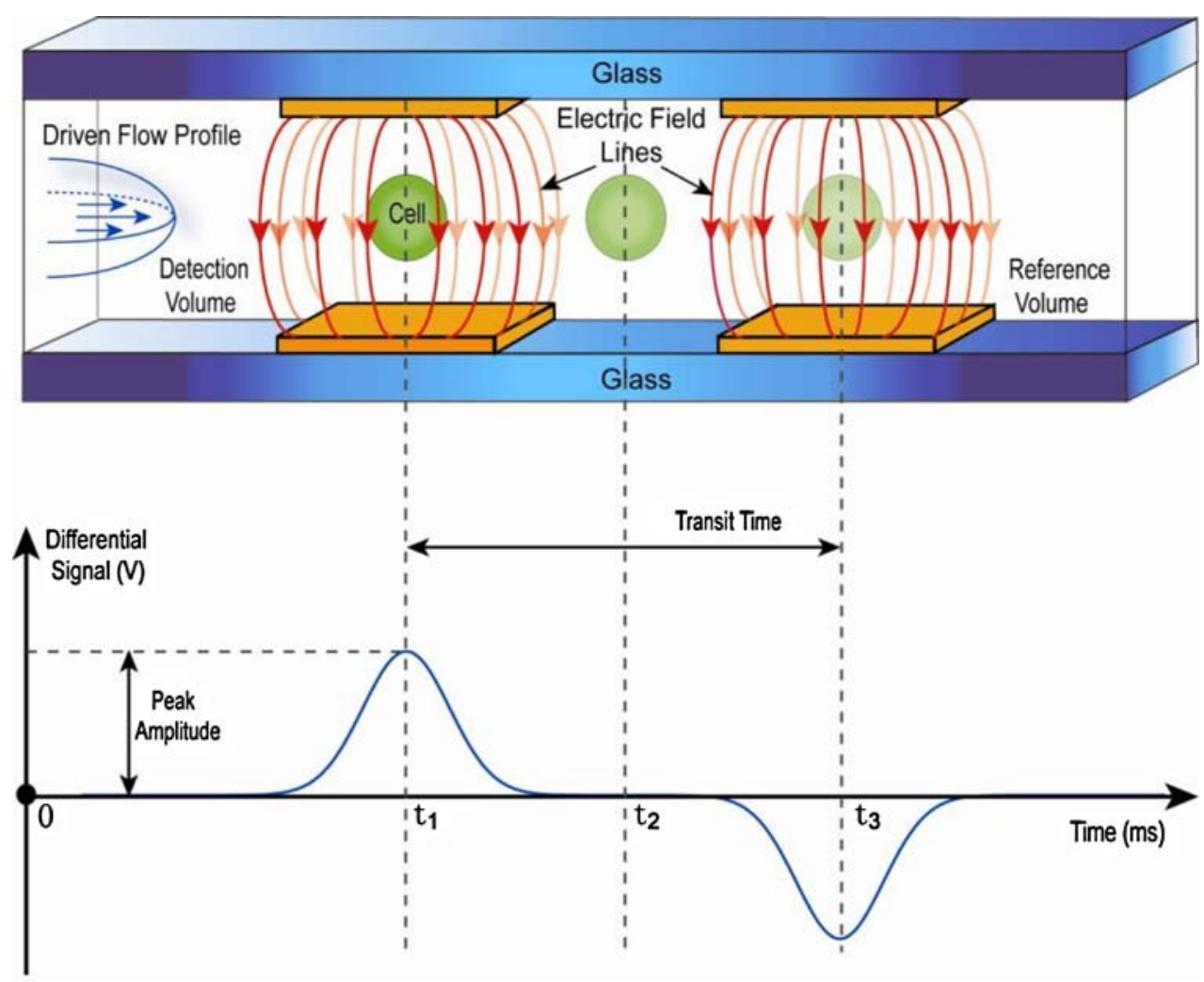


Fig. 2 Schematic diagrams for two different single cell impedance analysis systems. a AC discrete frequency system; measures impedance at single spot frequencies; b maximum length sequence system; performs broad-band measurements

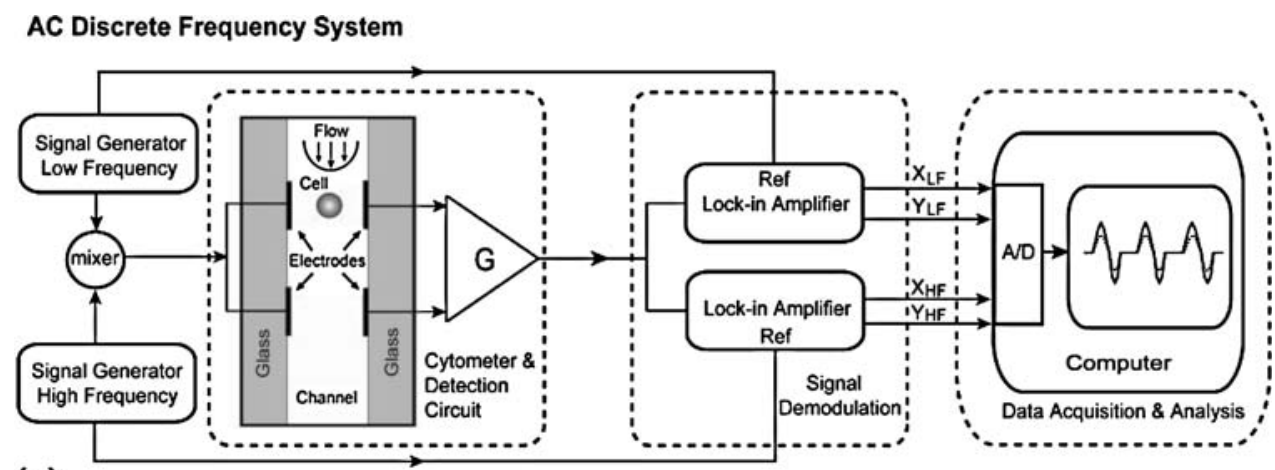

(a)

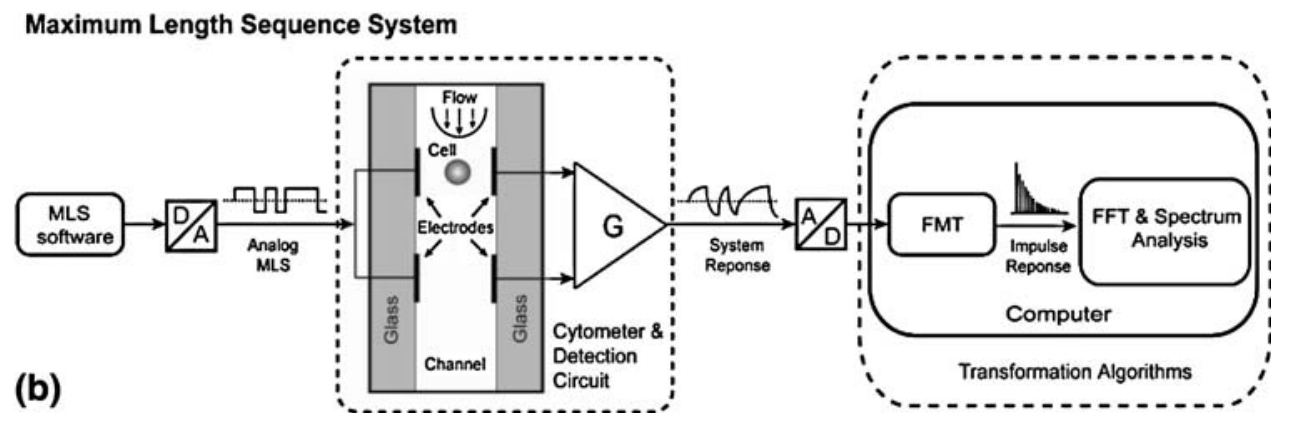

simultaneously measure up to eight frequencies at the price of complicated mixed-signal hardware instrumentation, Fuller et al. (2000).

\subsection{Maximum length sequence system}

In order to overcome the limitations of the $\mathrm{AC}$ discrete frequency system, we developed a multi-frequency impedance measurement system using maximum length sequences (MLS), Fig. 2b (Gawad et al. 2007; Sun et al. 2007b, c). MLS is a pseudo-random binary sequence (PRBS) and has a white-noise-like power density spectrum. The energy in the MLS is spread over a broad-band, enabling multi-frequency measurements to be made in one excitation period (ms) of MLS.

A digital MLS is generated in software and converted into an analog bipolar signal by D/A conversion. The analog MLS is applied to the cytometer and the differential signal measured by the same detection circuit. After A/D conversion, the analog system response is digitized and a Fast M-sequence Transform (FMT) algorithm used to obtain the impulse response of the system in the discrete time domain, followed by a Fast Fourier Transform to give the transfer-function in the frequency domain. The system has been used to measure the impedance spectrum latex beads and red blood cells (Sun et al. 2007b, c). With a tenth order MLS $\left(2^{10}-1=1,023\right.$ elements in one sequence) and a $1 \mathrm{MHz} \mathrm{A} / \mathrm{D}$ sampling rate, the impedance spectrum for a single cell can be measured within $1 \mathrm{~ms}$, with 512 discrete frequencies evenly distributed from $976.56 \mathrm{~Hz}$ to $500 \mathrm{kHz}$. However, a drawback of this method is a decrease in the SNR.

\section{Digital signal processing methods}

Two digital signal processing methods were used to analyse and filter the data: cross-correlation and adaptive filtering.

\subsection{Correlation method}

In signal processing, correlation measures the similarity of two signals. It is generally used to identify an unknown signal by comparing it with a known template function. The correlation between the two signals varies from 0 to 1 and provides a quantitatively measure of their similarity. If two signals are completely independent (no relationship), the Correlation Coefficient (CC) has a value of 0 . If two signals are identical the correlation is 1 .

The impedance signal from a flowing particle has a bipolar profile (Fig. 1). Correlation of this signal was performed using a template function consisting of two Gaussian functions, Fig. 3. Two point-spread Gaussian functions are positioned symmetrically around $t_{0}$, separated by a time $t_{\delta}$, with width $\sigma$ and amplitude scaling parameter $A$. The template function is therefore $f(t)$ :

$f(t)=A\left[\mathrm{e}^{g_{1}(t)}-\mathrm{e}^{g_{2}(t)}\right]$ 


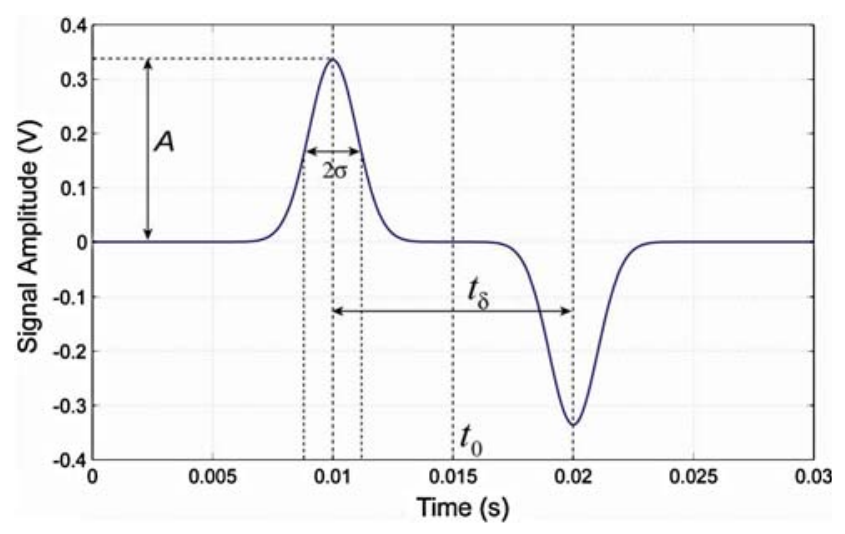

Fig. 3 A simulated trace of a differential impedance signal for a single particle, produced by a template fitting function, consisting of two Gaussian functions. There are four fitting parameters in this template function: central time moment, $t_{0}$; transit time, $t_{\delta}$; peak width control $\sigma$ and peak amplitude control $A$

with

$$
\begin{aligned}
A= & \frac{a}{\sqrt{2\left(1-\mathrm{e}^{\left(-t_{\delta}^{2} / 4 \sigma^{2}\right)}\right) \sqrt{\pi \sigma^{2}}}}, \\
& g_{1}(t)=-\frac{\left(t-t_{0}+t_{\delta} / 2\right)^{2}}{2 \sigma^{2}}, \quad g_{2}(t)=-\frac{\left(t-t_{0}-t_{\delta} / 2\right)^{2}}{2 \sigma^{2}}
\end{aligned}
$$

The correlation coefficient between two stochastic variables $X$ and $Y$, is defined as:

$\mathrm{CC}=\frac{E(X Y)-E(X) E(Y)}{\sqrt{E\left(X^{2}-E^{2}(X)\right)} \sqrt{E\left(Y^{2}-E^{2}(Y)\right)}}$

where $E$ is the expect value operator. The two variables $X$ and $Y$ correspond to the measured data stream and the sample stream from the template function, respectively.

\subsection{Adaptive filtering method}

The principles and applications of adaptive noise cancelling have been thoroughly addressed by Widrow et al. (1975). Adaptive digital filters have been widely used for background noise suppression and baseline correction in biomedical electronics. In electrocardiogram (ECG) recording, adaptive filtering has been used to remove power-line interference $(50$ or $60 \mathrm{~Hz})$ using a reference signal taken from the mains power supply (Widrow et al. 1975). Thakor and Zhu (1991) developed specialized filter structures for cancelling noise from various sources and the detection of arrhythmia in the ECG analysis. Narasimhan and Dutt (1996) used low pass filtering, followed by an adaptive predictive filter to cancel muscle artefacts in electroencephalograph (EEG) analysis. Pinto et al. (2002) developed a blood pressure monitoring system that used an adaptive interference canceller to remove noise and vibration from the measurement. Papezova (2003) used an adaptive digital filter to decrease the noise interference level in impedance changes in tissue due to blood flow. More recently, Sakuta et al. (2006) combined an adaptive filter and wavelet transform to remove noise from a magnetic signal measured by a superconducting quantum interference device.

In the above applications, a reference signal is recorded simultaneously with the primary signal from the object under test. The reference signal correlates with the noise in the primary signal and after adaptive filtering subtracted from the primary signal to give the noise-free output. However, in the single cell impedance measurement system, both pairs of electrodes are used for a differential measurement and a reference signal is not available. Widrow et al. (1975) proposed using a fixed delay in the raw data stream to serve as a reference signal, to extract a periodic signal from broad-band noise. This type of application is named an Adaptive Line Enhancer (ALE). Later Zeidler et al. (1978) analysed the steady-state behaviour of the ALE for stationary inputs consisting of multiple sinusoids buried in white noise. Treichler (1979) used an eigenvalue-eigenvector technique to quantify the convergence time and characteristics of the ALE. In our applications at least mean squared (LMS)-based ALE is used to remove background noise in the measurements and tested using the MLS data.

Figure 4 shows the structure of a LMS-based ALE (Widrow et al. 1975; Zeidler et al. 1978; Treichler 1979). The unfiltered signal (raw measurement data stream) consists of the narrow-band signals of interest (positivenegative peaks) together with broad-band noise, and is the primary channel of the ALE. The reference channel of the ALE is the delayed data stream of the unfiltered signal. Because of this delay, the broad-band component (noise) in the reference channel is de-correlated from the noise in the primary channel. When the output $y$ of the ALE, is subtracted from the signal in the primary channel $d$, the adaptive filter seeks to minimize the power of the feedback error, $e$, by recursively adjusting the weighting coefficients, $W_{(i, j)}(1 \leq i \leq L)$. The LMS algorithm for updating the weighting coefficients is:

$W_{(i, j+1)}=W_{(i, j)}+2 \eta e x_{(L-i+1, j)}$

where $L$ is the order of the adaptive filter (number of weighting coefficients). $W_{(i, j)}$ is a certain weighting coefficient at the time moment, $j$. $W_{(i, j+1)}$ is the corresponding weighting coefficient at the time moment, $(j+1) \cdot x_{(L-i+1, j)}$ is the delayed sample corresponding to the weighting coefficient $W_{(i, j)}$ at the time moment, $j$ (see Fig. 4).

The convergence parameter, $\eta$ can be evaluated (Semmlow 2004): 
Fig. 4 Diagram showing the structure of the adaptive line enhancer $(A L E)$ filter, which operates using the least mean square $(L M S)$ algorithm

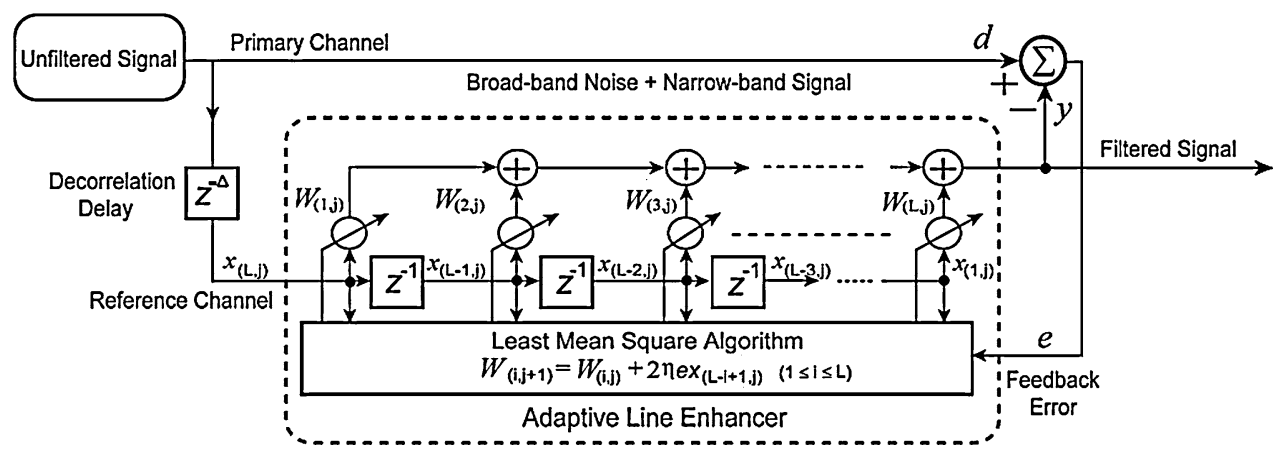

(4)

frequency and the MLS system using polystyrene beads. Beads of 5.49 and $7.18 \mu \mathrm{m}$ diameter (Bangs Laboratories Inc., USA) were suspended in the phosphate buffered saline (PBS) solution (conductivity $1.6 \mathrm{Sm}^{-1}$ ). For the MLS measurement, tenth order of MLS was used and the A/D sampling rate is $1 \mathrm{MHz}$. The correlation and the LMSbased ALE algorithms are implemented in Matlab ${ }^{\mathrm{TM}}$ (Mathworks Inc., USA).

\subsection{Correlation for particle identification}

The differential signal is made up of an in-phase (real) and out-of-phase (imaginary) component, exhibiting similar patterns (positive-negative peaks). Signal processing was applied to both signals, but in this paper we only show the in-phase (real) component. Figure 5 shows a differential signal for a $5.49 \mu \mathrm{m}$ bead and a $7.18 \mu \mathrm{m}$ bead in the continuous time course, measured at a frequency of $507 \mathrm{kHz}$ using the AC discrete frequency system. Also shown is the fitting template function used for each bead. The fitting template is set within a $60 \mathrm{~ms}$ time window. The values of the fitting parameters that give the best fits for the two beads are shown in Fig. 5, with $\mathrm{CC}=0.9765$
Fig. 5 Plot showing the correlation between the in-phase component of the measured impedance signal for two different sized beads. The signal is measured using the $\mathrm{AC}$ mixed-frequency system at a frequency of $507 \mathrm{kHz}$. The simulated trace (solid line) is the Gaussian fitting template. The correlation coefficient in this case is high, $\mathrm{CC}>0.95$

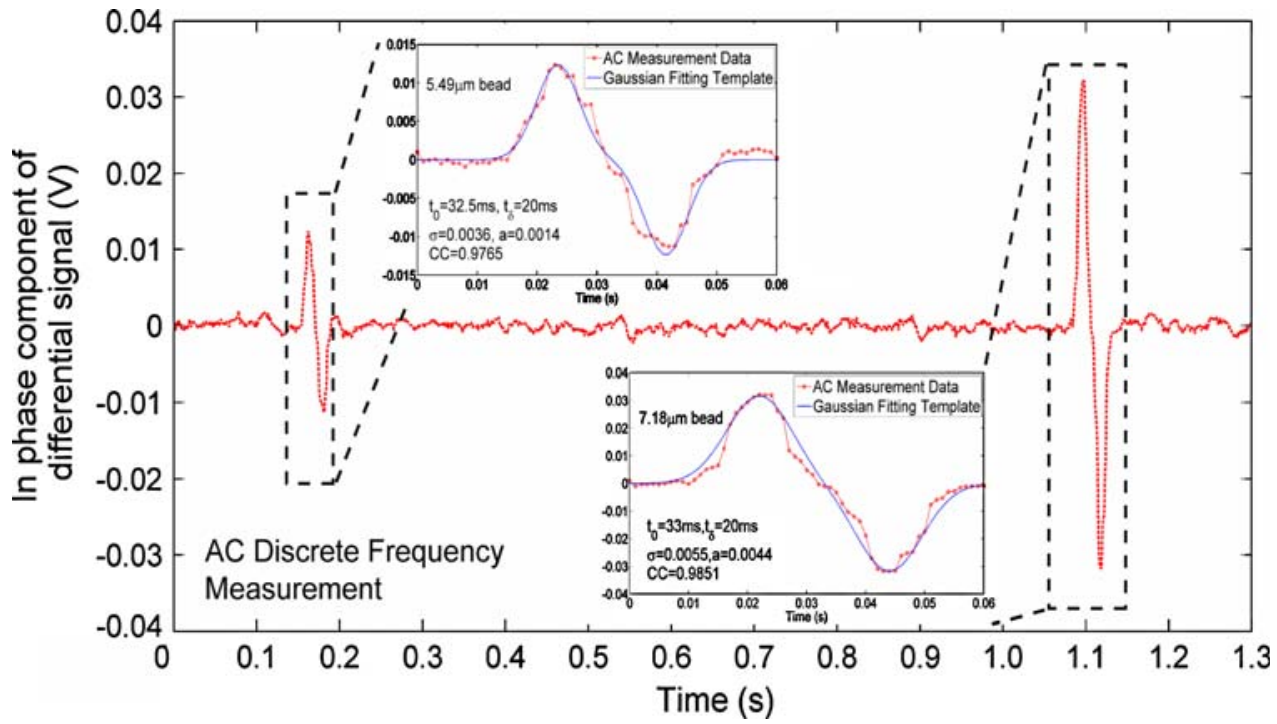


and $\mathrm{CC}=0.9851$ for the 5.49 and $7.18 \mu \mathrm{m}$ bead, respectively. The velocity of the bead is determined from the transit time $\left(t_{\delta}=20 \mathrm{~ms}\right)$, the electrode size $(20 \mu \mathrm{m})$ and gap $(20 \mu \mathrm{m})$, and is $2 \mathrm{~mm} / \mathrm{s}$. For automatic particle/cell counting, the system is set such that only signals with a correlation coefficient above a threshold (i.e. $\mathrm{CC} \geq 0.95$ ) are recorded.

Correlation can become problematic if the data is very noisy. Figure 6 shows the real part of the impedance signal for the same sizes of beads measured at $488.28 \mathrm{kHz}$ using the MLS system. The trend in the signal is evident but the noise is much worse. Performing a correlation in a $250 \mathrm{~ms}$ time window gives $\mathrm{CC}_{\mathrm{MG}}=0.8585$ for the $5.49 \mu \mathrm{m}$ bead and $\mathrm{CC}_{\mathrm{MG}}=0.9244$ for the $7.18 \mu \mathrm{m}$ bead. Applying the same threshold as previous $(C C \geq 0.95)$ shows that neither of the events will be recorded. The determination of an effective threshold value becomes difficult as the power of the noise increases because the correlation coefficient is reduced. This is particularly problematic at low frequencies because the excitation voltage is reduced due to the electrical double layer (Morgan et al. 2007).

\subsection{Adaptive filtering for noise reduction}

The MLS technique performs multi-frequency measurements in a short time window, but at the expense of degradation in the SNR because in general, periodic noise sequences (i.e. MLS) are extremely vulnerable to even slight time variances (Müller and Massarani 2001). The measurement system consists of cell flowing through a microchannel at high speed, and is not time-invariant system (Sun et al. 2007b). This time variance leads to a distortion in the signal that will appear as random noise superimposed on the impulse response of the system in the time domain, polluting the frequency domain data. Unlike the $\mathrm{AC}$ discrete frequency method, the system does not use lock-in amplifiers for noise rejection. The widely used synchronous averaging method does not improve the SNR in this case because the impedance spectrum of each cell is unique, and averaging methods cannot be used. Adaptive filtering therefore appears to be well suited for efficient removal of broad-band noise.

To demonstrate this, an adaptive filter was chosen with order $256(L=256)$ with a convergence gain empirically chosen to be $5 \%(\alpha=0.05)$. The reference channel is obtained by delaying the data stream by 100 samples. The influence of the filter order, convergence gain and delay length are discussed in the next section.

Applying the LMS-based ALE to the MLS data gives the results shown in Fig. 6. Comparing the unfiltered and filtered data shows effective noise removal. The correlation coefficient between the adaptive filtered data and the Gaussian fitting template is $0.9939\left(\mathrm{CC}_{\mathrm{AG}}=0.9939\right)$ for the $5.49 \mu \mathrm{m}$ bead and $0.9906\left(\mathrm{CC}_{\mathrm{AG}}=0.9939\right)$ for the $7.18 \mu \mathrm{m}$ bead. The improvement in the correlation coefficient implies that a high threshold value $(\mathrm{CC} \geq 0.95)$ can be used even in a noisy measurement.

The effect of adaptive filtering is pronounced at low frequencies. Figure 7 a shows the MLS signal (dashed line) measured at $39.063 \mathrm{kHz}$ for the same two beads presented in Fig. 6 (measured at $488.28 \mathrm{kHz}$ ). The signal from these two beads is very noisy, so that it becomes impossible to use correlation. However, adaptive filtering efficiently recovers the data, solid line Fig. 7a. This data can now be correlated against the Gaussian fitting templates in the same time window $(250 \mathrm{~ms})$ as used for the high frequency data (Fig. 6). Apart from the amplitude of the templates, other fitting parameters are exactly the same as previous,
Fig. 6 Plot showing the real part of the differential signal for two different sized beads measured using MLS, and plotted for a single frequency of $488.28 \mathrm{kHz}$. Also shown is the simulated trace of the Gaussian fitting template, together with the adaptive filtered data. The correlation efficient is improved after adaptive filtering

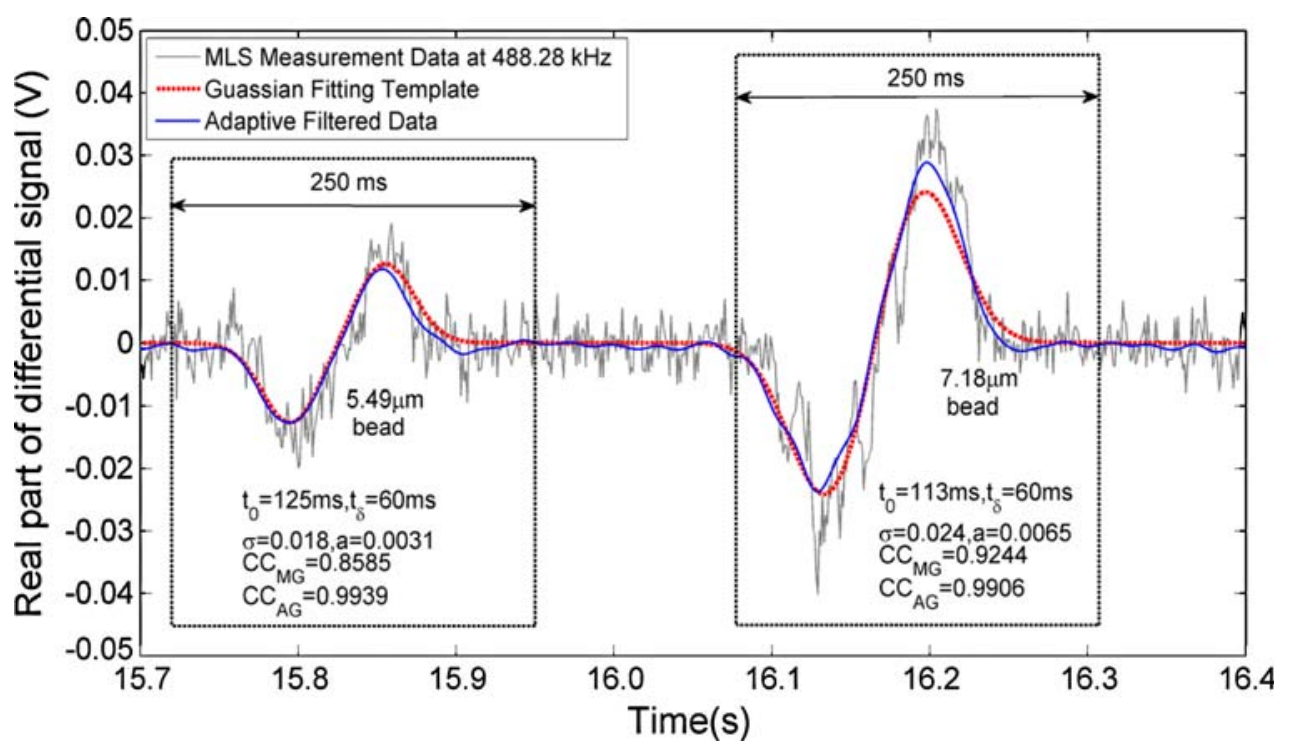


Fig. 7 a Plot showing the MLS data for the same two beads as plotted in Fig. 6, but at $39.063 \mathrm{kHz}$. At this frequency, it is extremely difficult to identify the signal from the beads in the raw data (dashed line). However, adaptive filtering recovers the signal. b, c The correlation between the adaptive filtered data and the Gaussian fitting templates, significantly increasing the correlation coefficient

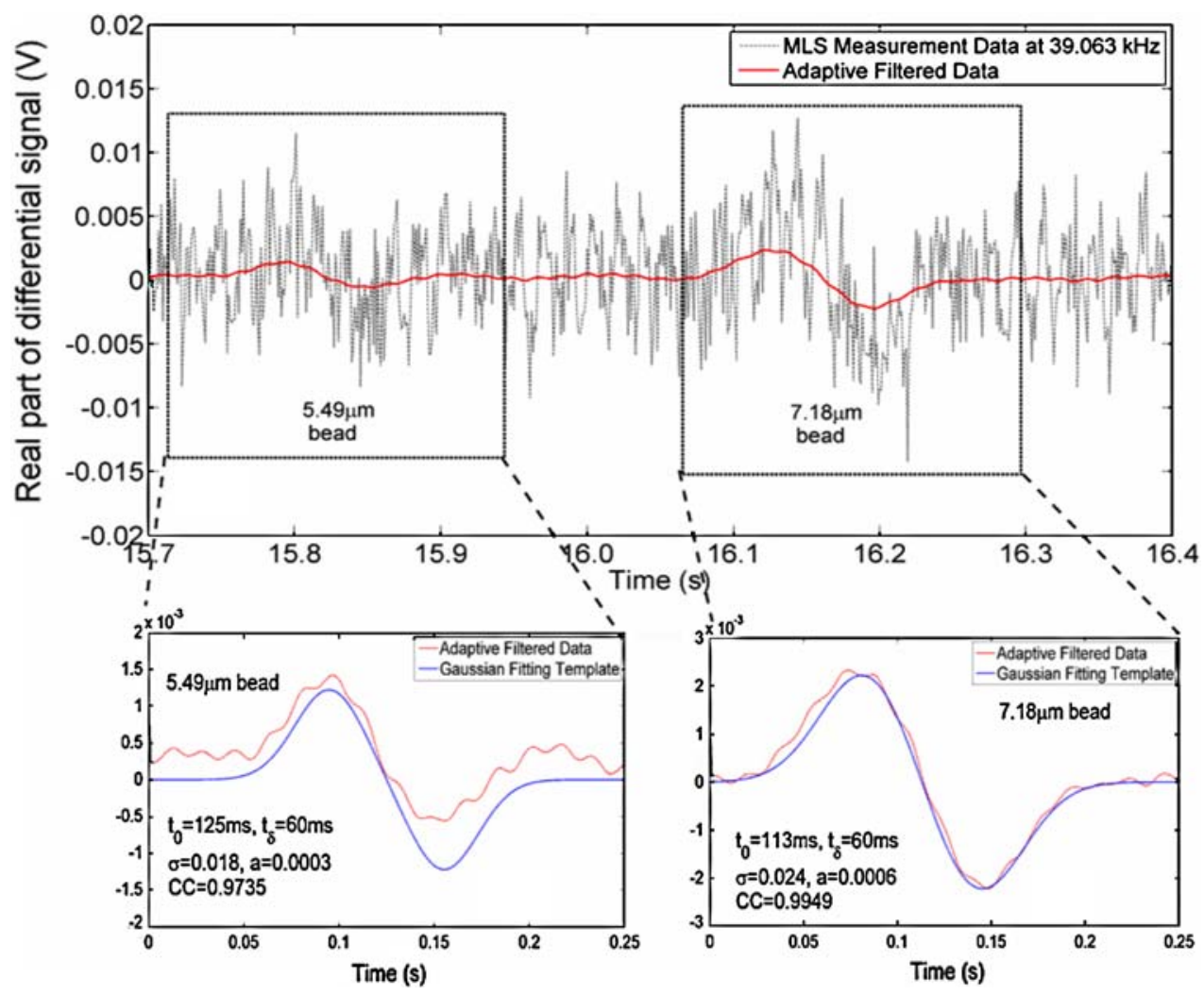

confirming that the same two beads were measured. For MLS measurements, the transit time of a particle should be much longer than the period of a single sequence, reducing the time variance in the measurement. In this data, the particle velocity is approximately three times less than in the AC discrete frequency measurements. Note that at low frequencies $(39.063 \mathrm{kHz})$, the signal is a positive-negative peak, while at high frequencies $(488.28 \mathrm{kHz})$, the pattern is reversed because of the transfer function of the differential electronic circuitry for signal measurement.

The increase in SNR was calculated from the ratio of the power between a single bead signal, $P_{\text {bead }}$, and the background noise, $P_{\text {noise }}$, (Eq. 5):

$\mathrm{SNR}(\mathrm{dB})=10 \log _{10}\left(\frac{P_{\text {bead }}}{P_{\text {noise }}}\right)$

Using the $5.49 \mu \mathrm{m}$ bead (Figs. 6, 7) as an example. Before adaptive filtering, the SNR is $9.23 \mathrm{~dB}$ (at $488.28 \mathrm{kHz}$ ); after filtering, SNR increases to $27.33 \mathrm{~dB}$. At $39.063 \mathrm{kHz}$, the SNR is $-5.13 \mathrm{~dB}$, indicating that the noise power exceeds signal from the beads. After filtering, the SNR increases to $15.45 \mathrm{~dB}$. Therefore adaptive filtering improves the SNR by approximately $20 \mathrm{~dB}$.

\subsection{Savitzky-Golay filtering}

The Savitzky-Golay (S-G) method (Savitzky and Golay 1964) is probably the most commonly used method for data smoothing and differentiation and is widely used in analytical chemistry. It is a polynomial filter and calculates the smoothed value for every point in a data stream, based on a series of convolution weighting factors. The S-G method has been used (Sun et al. 2007b, c) to process MLS data, returning a spectrum that was in agreement with $\mathrm{AC}$ discrete frequency measurements and PSpice circuit simulations. However, the filtered signal has noise with a pseudo-periodic sinusoidal form (see Fig. 5a, b in Sun et al. 2007c).

The degree of noise reduction and signal enhancement depends on the order of the polynomial and the length of the filter. Figure $8 \mathrm{a}$ shows a data stream processed with $\mathrm{S}-\mathrm{G}$ filters of different order and length. The higher order $S-G$ filter preserves the peak values but has poor overall noise reduction; the pseudo-periodic filtered noise is observed. A longer $\mathrm{S}-\mathrm{G}$ filter has better noise reduction, the filtered noise no longer has a pseudo-periodic, but the peak value of the signal is reduced. An optimal polynomial order and filter length is difficult to choose. The S-G filter smoothes the data by replacing each data point with a local average of the surrounding data points (Mittermayr et al. 1997). Because it is not locally adaptive, optimisation requires performing tests with different combinations of polynomial order and filter length on the same data stream (Barak 1995).

In the ALE mode, adaptive filtering has three parameters: filter order, convergence gain and delayed samples, one more parameter than the S-G filter. However, the method has better inherent stability than the $\mathrm{S}-\mathrm{G}$ filter. As shown in 
Fig. 8 a Plot showing the MLS data and the Savitzky-Golay filter output for different filter orders and lengths. b Plot showing MLS data and the adaptive filter output for different filter orders, convergence gain and sample delayed
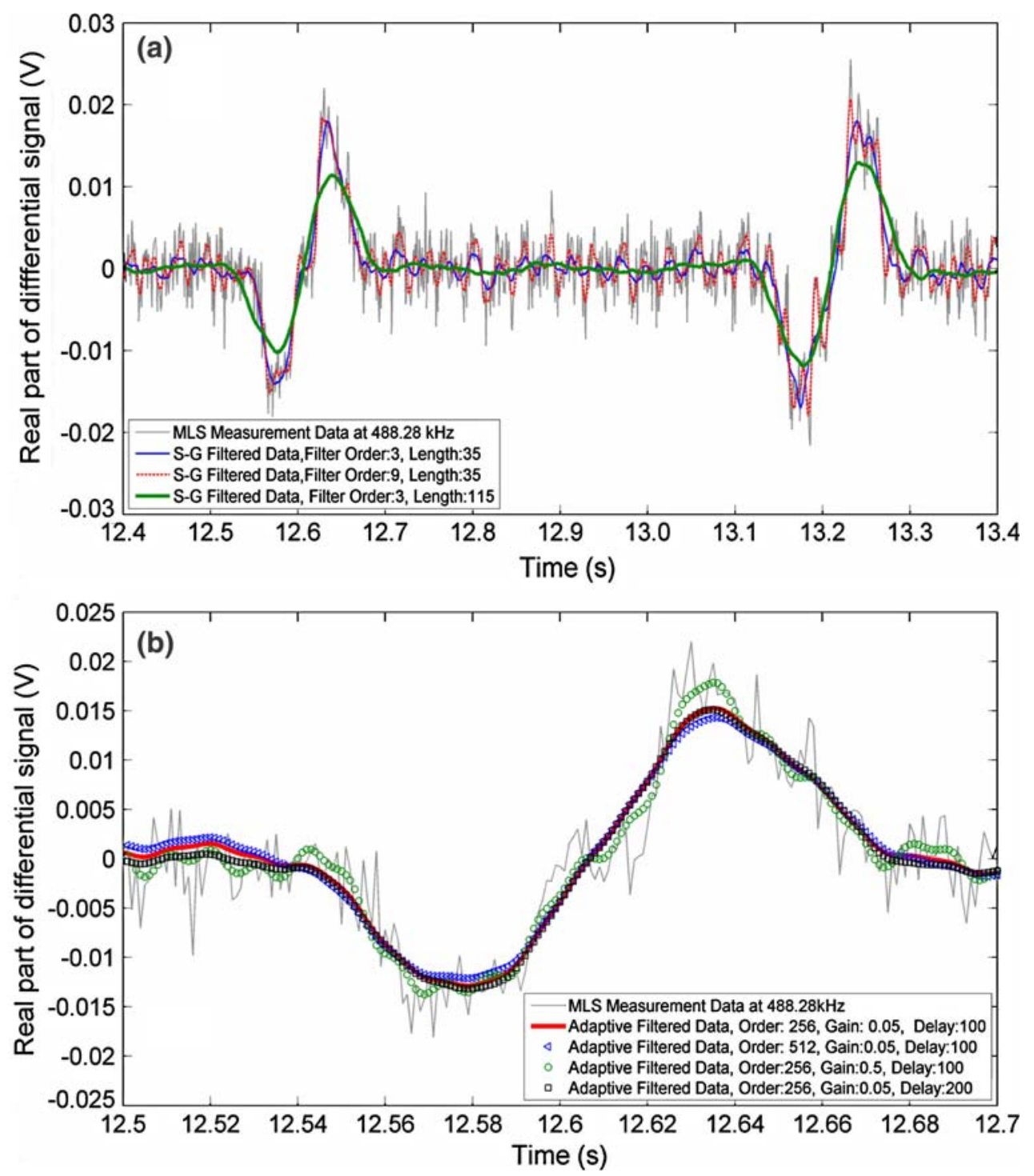

Fig. 4, the adaptive filter operates by updating the weighting coefficients depending on the property of the signals in the primary and reference channels. The LMS algorithm uses a recursive gradient method known as the steepestdescent method (Semmlow 2004) to find the weighting coefficients that produce minimum sum of squared error. ALE is also regarded as a self-tuning narrowband filter. Figure $8 \mathrm{~b}$ shows how the filtered signal varies with choice of filter order, convergence gain and number of delayed samples (enlarged to show the small differences). The determination of the setting parameters of the adaptive filtering has a wider freedom than for the $\mathrm{S}-\mathrm{G}$ filter.

\section{Conclusions}

In this paper, we show how digital signal processing methods can be used to extract noisy signals from in single particle impedance micro-cytometry. The correlation method can be used to identify and count events where the SNR is already good, as in a system with lock-in amplifiers. For a noisy environment/measurement system (e.g. MLS), the ALE removes the noise, improving the SNR of the system by about $20 \mathrm{~dB}$. After filtering, the correlation method can be subsequently applied to the filtered data stream to indentify/count the flowing particles. The superiority of the adaptive filtering method over the commonly used Savitzky-Golay method has been demonstrated in terms of the inherent stability and improved performance. The ALE method is expected to find applications for detection of weak signals in a number of areas of biotechnology and biomedicine.

Acknowledgments This work was partly funded by the University of Southampton, Life Science Initiative (LSI) and Philips. The authors would like to thank Mr. Rong Zhang for valuable discussions. 


\section{References}

Ayliffe HE, Frazier AB, Rabbitt RD (1999) Electric impedance spectroscopy using microchannels with integrated metal electrodes. IEEE J MEMS 8:50-57

Barak P (1995) Smoothing and differentiation by an adaptive-degree polynomial filter. Anal Chem 57:2758-2762

Cheung K, Gawad S, Renaud Ph (2005) Impedance spectroscopy flow cytometer: on-chip label-free cell differentiation. Cytometry Part A $65 \mathrm{~A}: 124-132$

Coulter WH (1956) High speed automatic blood cell counter and cell analyzer. Proc Natl Electron Conf 12:1034-1040

Cho YH, Yamamoto T, Sakai Y, Fujii T, Kim B (2006) Development of microfluidic device for electrical/physical characterization of single cell. J Microeletromech Syst 15:287-295

Fuller CK, Hamilton J, Ackler H, Krulevitch P, Boser B, Eldredge A, Becker F, Yang J, Gascoyne P (2000) Microfabricated multifrequency particle impedance characterization system. Proc $\mu$ TAS 2000:265-268

Gawad S, Cheung K, Seger U, Bertsch A, Renaud Ph (2004) Dielectric spectroscopy in a micromachined flow cytometer: theoretical and practical considerations. Lab Chip 4:241-251

Gawad S, Schild L, Renaud Ph (2001) Micromachined impedance spectroscopy flow cytometer for cell analysis and particle sizing. Lab Chip 1:76-82

Gawad S, Sun T, Green NG, Morgan H (2007) Impedance spectroscopy using maximum length sequences (MLSA): application to single cell analysis. Rev Sci Instrum 78:054301

Holmes D, Sandison ME, Green NG, Morgan H (2005) On-chip highspeed sorting of micron-sized particles for high-throughput analysis. IEE Proc Nanobiotechnol 152:129-135

Jang LS, Wang MH (2007) Microfluidic device for cell capture and impedance measurement. Biomed Microdevices 9:737-743

Mittermayr CR, Frischenschlager H, Rosenberg E, Grasserbauer M (1997) Filtering and integration of chromatographic data: a tool to improve calibration? J Anal Chem 358:456-464

Malleo D, Nevill JT, Carlo DD, Lee LP, Morgan H (2007) Examining cytotoxic effects on single cells using an impedance spectroscopy platform. Proc $\mu$ TAS 2:1083-1085

Morgan H, Holmes D, Green NG (2006) High speed simultaneous single particle impedance and fluorescence analysis on a chip. Curr Appl Phys 6:367-370

Morgan H, Green NG (2003) AC electrokinetics: colloids and nanoparticles. Research Studies Press, Baldock

Morgan H, Sun T, Holmes D, Gawad S, Green NG (2007) Single cell dielectric spectroscopy. J Phys D Appl Phys 40:61-70
Müller S, Massarani P (2001) Transfer-function measurement with sweeps. JAES 443-471

Narasimhan SV, Dutt DN (1996) Application of LMS adaptive predictive filtering for muscle artifact (noise) cancellation from EEG signals. Comput Elect Eng 22:13-30

Pinto L, Dhanantwari A, Wong W, Stegriopoulos S, Maris M (2002) Blood pressure measurement in noise intensive environments using adaptive interference cancellation. Ann Biomed Eng 30:657-670

Papezova S (2003) Signal processing of bioimpedance equipment. Sens Actuators B 95:328-335

Savitzky A, Golay MJE (1964) Smoothing and differentiation of data by simplified least squares procedures. Anal Chem 36:16271639

Sakuta K, Mizoguti K, Setoguchi A, Itozaki H (2006) System noise cancellation by digital signal processing for SQUID measurement. Superconductor Sci Technol 19:S407-S410

Sun T, Green NG, Gawad S, Morgan H (2007a) Analytical electric field and sensitivity analysis for two microfluidic impedance cytometer designs. IET Nanobiotechnol 1:69-79

Sun T, Gawad S, Bernabini C, Green NG, Morgan H (2007b) Broadband single cell impedance spectroscopy using maximum length sequences: theoretical analysis and practical considerations. Meas Sci Tech 18:2859-2868

Sun T, Holmes D, Gawad S, Green NG, Morgan H (2007c) High speed multi-frequency impedance analysis for single particles in a microfluidic cytometer using maximum length sequences. Lab Chip 7:1034-1040

Semmlow JL (2004) Biosignal and biomedical image processing Matlab-based applications. Marcel Dekker, NewYork

Treichler JR (1979) Transient and convergent behavior of the adaptive line enhancer. IEEE Trans Acoust Speech Signal Process ASSP 27:53-62

Thakor NV, Zhu YS (1991) Applications of adaptive filtering to ECG analysis: noise cancellation and arrhythmia detection. IEEE Trans Biomed Eng 38:785-794

Wang YN, Kang Y, Xu D, Chon CH, Barnett L, Kalams SA, Li D, Li D (2008) On-chip counting the number and the percentage of CD4+ T lymphocytes. Lab Chip 8:309-315

Widrow B, Glover JR, McCool JM, Kaunitz J, Willams CS, Hearn RH, Zeidler JR, Dong E, Goodlin RC (1975) Adaptive noise cancelling: principles and applications. Proc IEEE 63:16921716

Zeidler JR, Satorius EH, Chabries DM, Wexler HT (1978) Adaptive enhancement of multiple sinusoids in uncorrelated noise. IEEE Trans Acoust Speech Signal Process ASSP 26:240-254 